\title{
Implementation of Oracle RAC on Oracle Cloud
}

\author{
Skant Gupta \\ Oracle DBA, Etisalat \\ Dubai
}

\author{
Stuti Kaushal \\ Senior System Engineer \\ Infosys
}

\begin{abstract}
Today's world is all about technology our life is contingent upon technology. Little change in technology makes huge differences on today's business. Cloud computing is a virtualized compute power and storage delivered via platformagnostic infrastructure of abstracted hardware and software accessed over the internet.

In this paper is equipped with all the details about cloud computing. It also includes brief overview about oracle database, Oracle RAC and oracle cloud computing. This paper is focused on advantages of cloud computing. It also focuses on the steps to setup Oracle RAC on cloud.
\end{abstract}

\section{Keywords}

Oracle RAC, Oracle Cloud, Database as a service

\section{INTRODUCTION}

Data is the most important element of any alliance. Security of data is of utmost importance. Data plays a very essential role in each $n$ every department of an organization like IT department, R\&D department, HR department, Finance department, sales departments etc. In this hi-tech world there are lots of options available in market thereby allowing people to choose any database according to their budget, volume of data, quality etc. Some of the example of most saleable database includes Oracle, DB2, MySQL, SQLite, SQL Server and Sybase.

Oracle Database is the first RDBMS database designed for enterprise grid computing, the most flexible and cost effective way to manage information and applications by Oracle Corporation on 1977. Oracle database is amongst one of the best option for any organization because of its Reliability, Functionality, Customer Satisfaction and Flashback Technology, consistency, isolation, atomicity and durability.

The database has logical structures and physical structures. Because the physical and logical structures are separate, the physical storage of data can be managed without affecting the access to logical storage structures.

\section{ORACLE RAC}

When we talk about oracle database that is related to single database only. Data is increasing day by day. Due to increment of data organizations felt the need to switch in to Oracle RAC. RAC stands for Oracle Real Application Clusters. Oracle Clusterware is a portable cluster management solution that is integrated with Oracle Database. Oracle Clusterware is also a required component for using Oracle RAC. In addition, Oracle Clusterware enables both singleinstance Oracle databases and Oracle RAC databases to use the Oracle high-availability infrastructure. Oracle Clusterware enables us to create a clustered pool of storage to be used by any combination of single-instance and Oracle RAC databases.

\section{RACEVOLUTION}

Oracle 8i OPS implementation brought in many outstanding improvements done by oracle Corporation. The extraordinary new feature was the launch of cache fusion technology. Cache fusion, as appears earlier, In Rac cache, or SGA, multiple instances which coordinate the buffers and manages the database access. Oracle $8 \mathrm{i}$ (OPS) introduced the initial phase of cache fusion. The data blocks were transferred from the SGA of one instance to the SGA of another instance without the need to write the blocks to disk RAC mainly. The partial implementation of cache fusion in Oracle 8i could help only in certain conditions.

\section{THE BENEFITS OF REAL} APPLICATION CLUSTERS

- Lower Overall Cost of Ownership

- Expanded Scalability

- High Availability

- Transparency

- Buffer Cache Management

- $\quad$ Row Locking

- Recovery Manager, Online Backups, and Archiving

- Ability to spread CPU load across multiple servers

- Continuous Availability / High Availability (HA)

- RAC can take advantage of larger SGA sizes

- Scalability

- While choosing RAC for used as a cost savings solution

- Never choose RAC to scale

\section{WHAT IS CLOUD COMPUTING?}

As we know technology changes day by day which is making our life easy? New inventions occur every day. Cloud Computing is in trend. We can have some popular cloud computing products like Oracle Cloud, Amazon Web Service, Google Cloud Platform Services, and Microsoft Azure etc.

The computing which depends on sharing computing resources rather than having local servers or personal devices to handle applications are referred to as cloud computing. Cloud computing is akin to grid computing which is unused processing cycles of all computers in a network are harnesses to solve problems too intensive for any stand-alone machine. We have big advantage in cloud computing that it is based on shared, elastic resources delivered to users in a self-service, metered manner using web technologies.

\section{WHAT IS ORACLE CLOUD?}

Previous topics were based on RDBMS, Oracle RAC. Let us now learn about latest technology of Oracle Corporation in 
database. $12 \mathrm{c}$ is a latest version in oracle database. $\mathrm{C}$ stands for cloud computing Oracle Cloud is the industry's broadest and most integrated public cloud. Oracle provides best-inclass services across software as a service (SaaS), platform as a service (PaaS), and infrastructure as a service (IaaS), and even lets you put Oracle Cloud in your own data center. Oracle Cloud succor organizations drive innovation and business by increasing business agility, lowering costs, and reducing IT complexity.

\section{Advantages of Oracle Cloud Computing}

1. Oracle Cloud provides maximum performance and value to businesses.

2. In Oracle Cloud we have new feature that we can run Oracle's modern Fusion Applications out of the box.

3. Social relationship platform for engaging customers, partners, and employees easily can make in cloud computing.

4. Social brilliance and inbound insights are part of it.

5. One new feature of Optimizes Platform-as-aService integration with Oracle Cloud applications is available in oracle cloud.

6. Customers have more independence and decisionmaking in hands of customers.

7. Customers world-class security and architecture are on priority of cloud computing.

8. Customers can create itineraries for upgrades according their comforts.

9. Release of money and time for customers via completeness of the end-to-end solution.

10. Systems integrators function is available for Frees customers.

\section{CREATION OF ORACLE RAC DATABASE IN THE CLOUD}

Let us learn the procedure to create an Oracle RAC Database in the cloud. To do this, we enter with our account for Oracle cloud services, go to the "Oracle Database Cloud Service" page and create a new service.

We provide the Service name then we choose "Oracle Database Cloud Service" as Subscription Type, also provide the SSH public key.

Step 1: Explains about the selection of the database's release as shown in the figure. Select a database "release" and we chose "Enterprise Edition - Extreme Performance" as Software Edition. Then we continue with a click on "Next".

Elaborate about we provide an Administration Password Which is used as your sys password, DB name (SID) with respective (PDB Name) and mark the "Database Clustering with RAC" checkbox. Then we continue with a click on "Next".
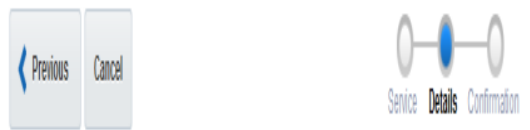

\section{Senicentalls}

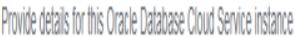
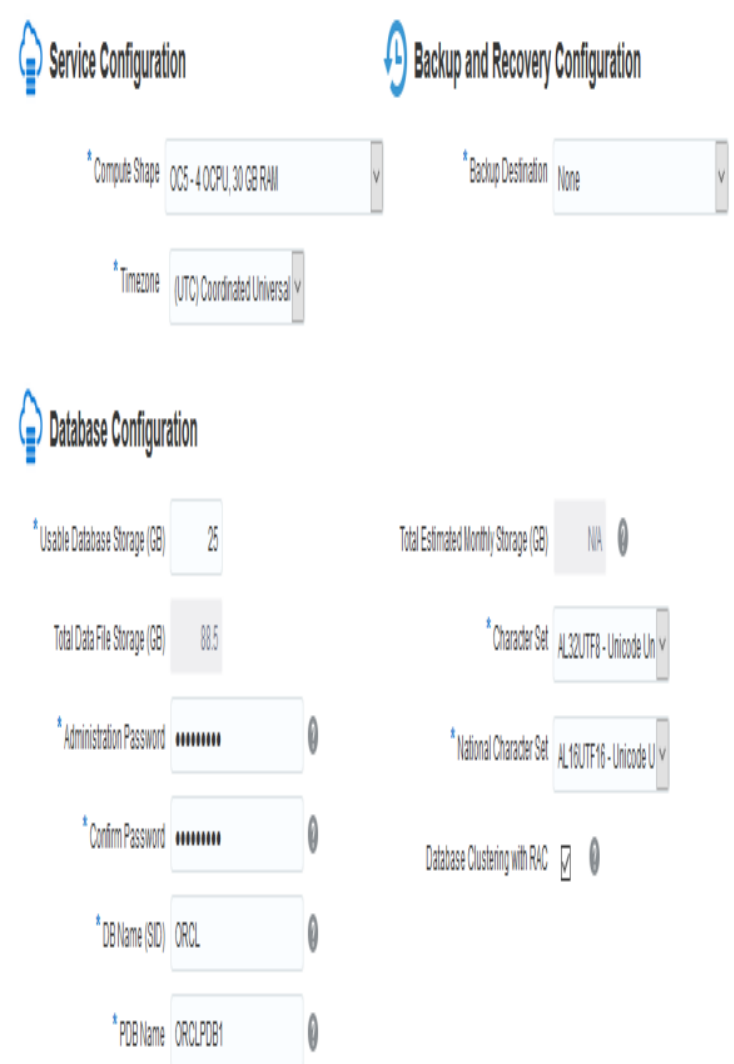

Fig.1. Explains about the administrator's password

Step 2: Finally, we check the configuration and click on "Create" to create Oracle RAC database on cloud. 

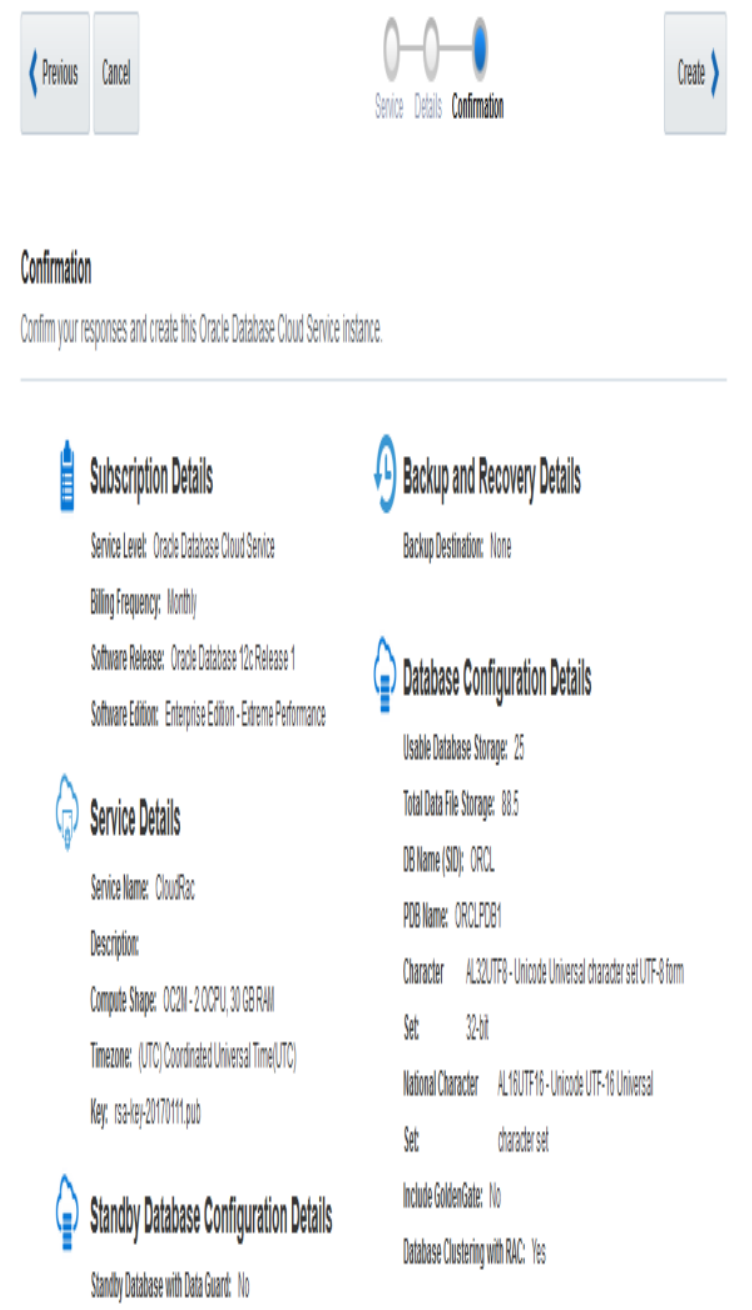

Fig. 3 Creation Summary

After a few minutes, Oracle RAC database is created successfully.

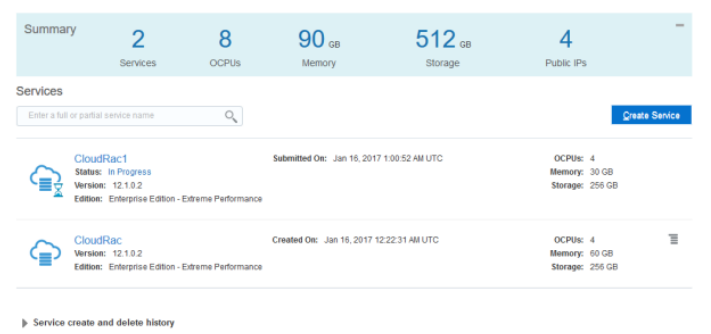

Fig 4. Main Service console

Step 4: we click on the service name (CloudRac) to open the main page of both instances of RAC.

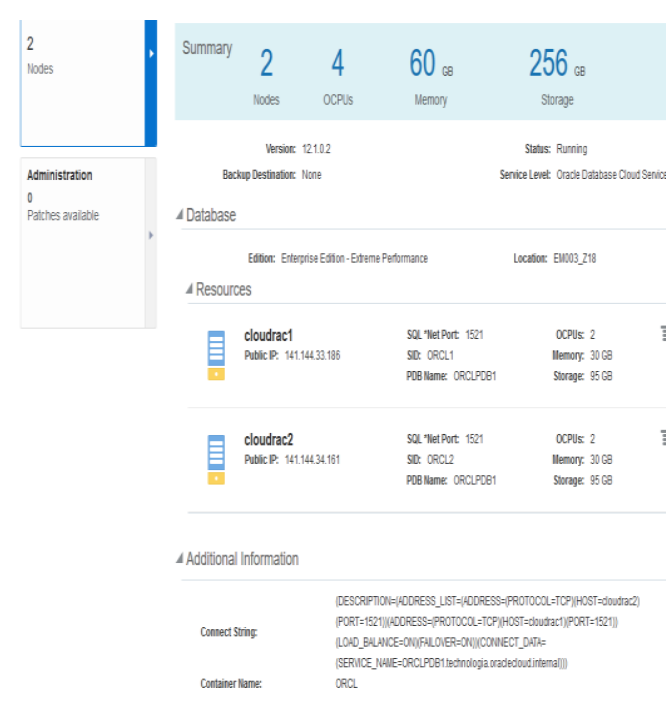

Fig.4. Opening of instance with RAC

Step 5: Now we are going to open two different instances of the "Putty" executable and we are going to connect SSH to both machines.

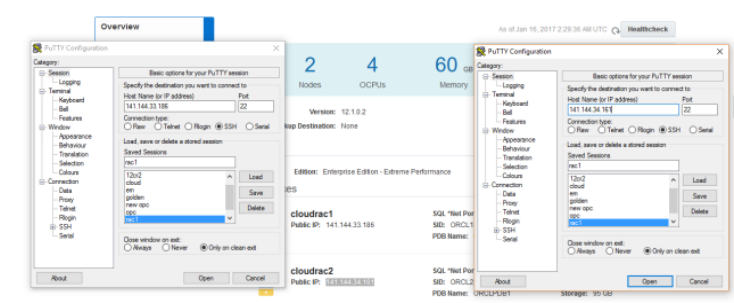

Fig.5. Connection with putty

Step 6: We connect to both instances and check the pmon on each server.

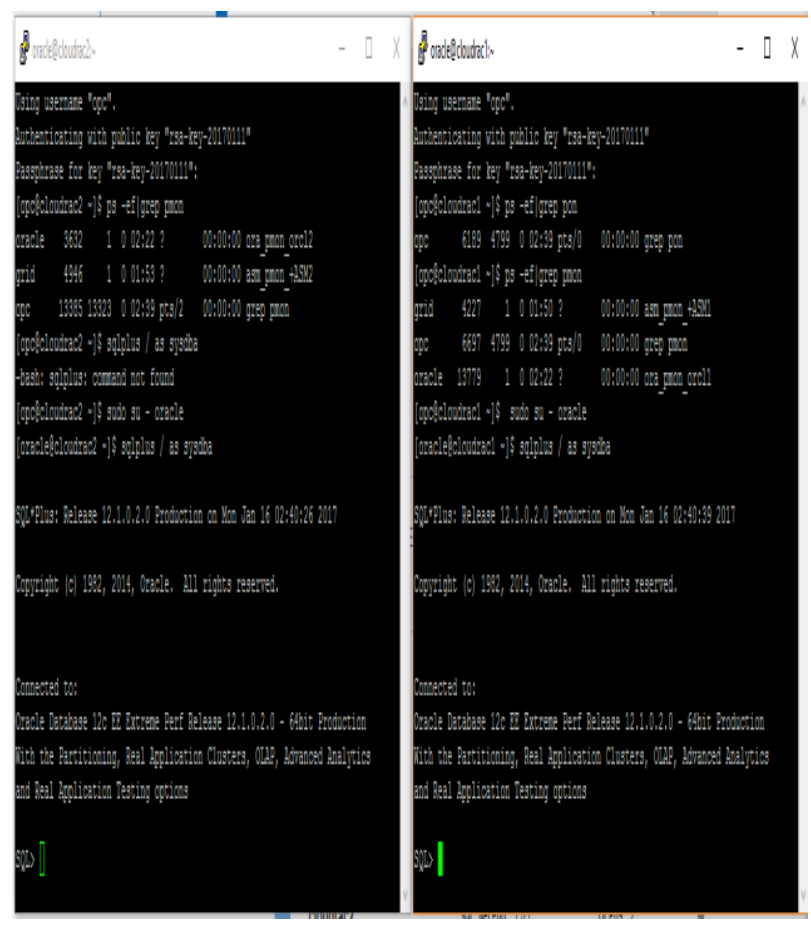

Fig.6. Check of pmon process 
Step 7: We check number of instance in Oracle RAC database as shown below.

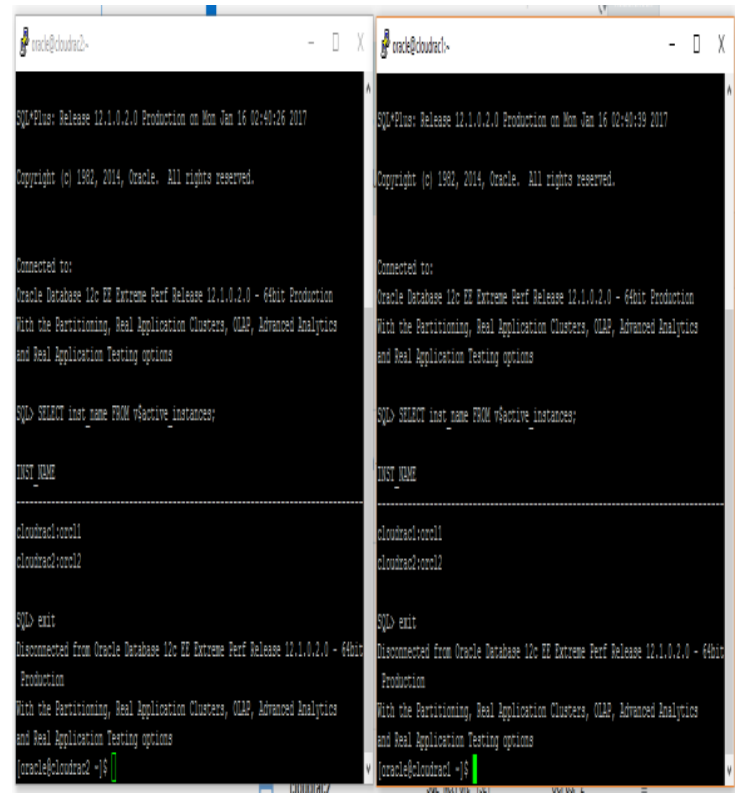

Fig. 7 checking number of instance

Step 8: We can cross verify the status of Oracle Database by using "srvctl" utility.

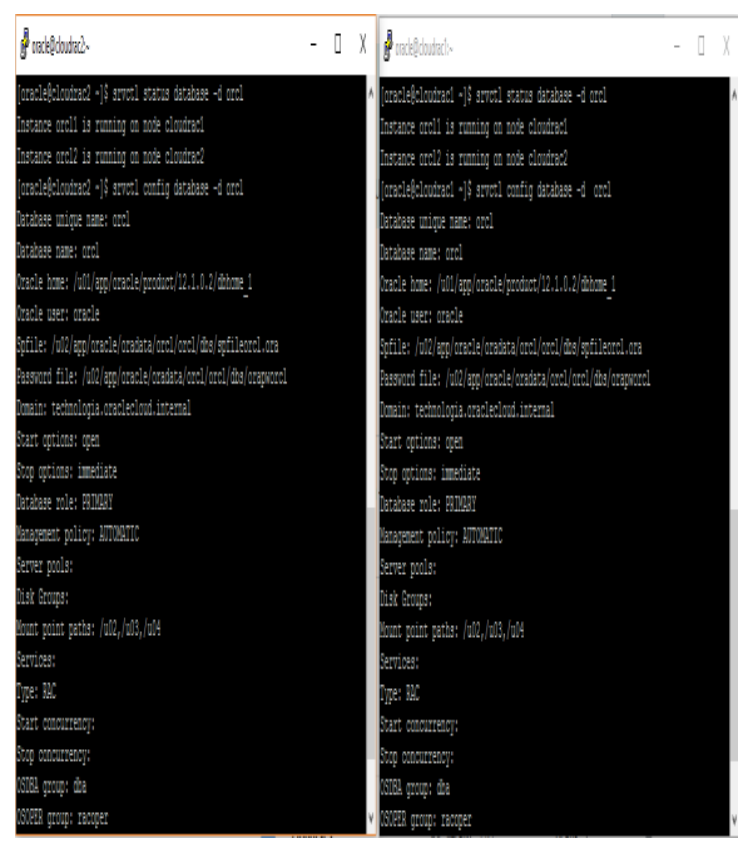

Fig.8. cross verify the status of Oracle Database

Step 9: Now we are connecting to "grid" user and check the clusterware resource by using "crsctl" utility.

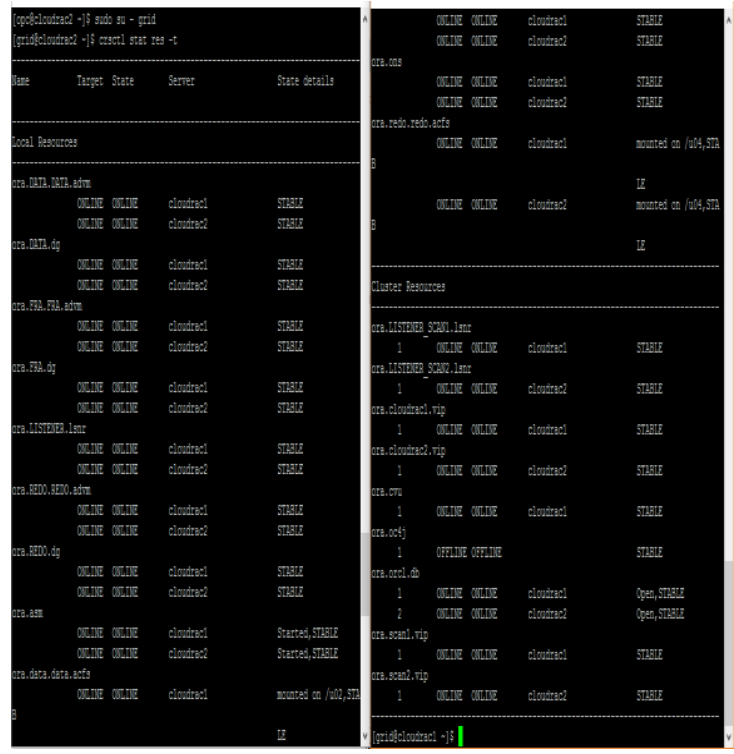

Fig.9. Connection with "grid"

Step 10: Now we can connect to PDB database in each instance of Oracle RAC.

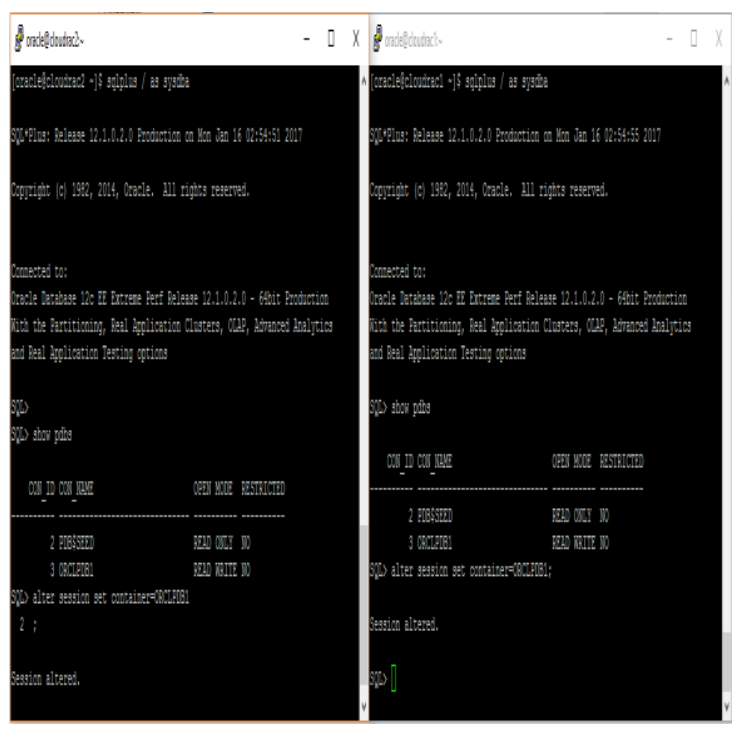

Fig.10. Connection with PDB

\section{CONCLUSION}

It is very easy to create a RAC database in the cloud. Moreover monitoring and troubleshooting is also not much difficult.

\section{REFERENCES}

[1] http://docs.oracle.com/en/cloud/paas/database-dbaascloud/csdbi/index.htm

[2] https://docs.oracle.com/en/cloud/paas/database-dbaascloud/csdbi/index.htm

[3] https://docs.oracle.com/en/cloud/paas/database-dbaascloud/csdbi/use-rac-this-service.html

[4] https://docs.oracle.com/cd/E11882_01/rac.112/e41960/to c.htm 